\title{
Human induced pluripotent stem cell-based microphysiological tissue models of myocardium and liver for drug development
}

\author{
Anurag Mathur',2, Peter Loskill',2, SoonGweon Hong1, Jae Young Lee ${ }^{1,3}$, Sivan G Marcus', Laure Dumont4, \\ Bruce R Conklin ${ }^{5,6}$, Holger Willenbring ${ }^{4,7}$, Luke P Lee ${ }^{* 1,8}$ and Kevin E Healy ${ }^{* 1,2}$
}

\begin{abstract}
Drug discovery and development to date has relied on animal models, which are useful but are often expensive, slow, and fail to mimic human physiology. The discovery of human induced pluripotent stem (iPS) cells has led to the emergence of a new paradigm of drug screening using human and disease-specific organ-like cultures in a dish. Although classical static culture systems are useful for initial screening and toxicity testing, they lack the organization of differentiated iPS cells into microphysiological, organ-like structures deemed necessary for high-content analysis of candidate drugs. One promising approach to produce these organ-like structures is the use of advanced microfluidic systems, which can simulate tissue structure and function at a micron level, and can provide high-throughput testing of different compounds for therapeutic and diagnostic applications. Here, we provide a brief outline on the different approaches, which have been used to engineer in vitro tissue constructs of iPS cell-based myocardium and liver functions on chip. Combining these techniques with iPS cell biology has the potential of reducing the dependence on animal studies for drug toxicity and efficacy screening.
\end{abstract}

\section{Introduction}

Current methods to evaluate drug safety and efficacy are costly, inefficient, and rely on nonhuman animal models. The human body is an extremely complex system and

*Correspondence: kehealy@berkeley.edu; Iplee@berkeley.edu 'Department of Bioengineering, B131 Stanley Hall, University of California at Berkeley, Berkeley, CA 94720-1762, USA

Full list of author information is available at the end of the article even advanced animal models do not fully mimic the human physiology or recapitulate human disease states. The data obtained with animal models therefore cannot necessarily be extrapolated to human subjects. For example, in 2004 the US Food and Drug Administration reported that 92 out of every 100 drugs that successfully passed animal trials subsequently fail human trials [1]. Moreover, out of every 10,000 compounds that go through research and development, approximately 5 to 10 drugs make it to the clinical trials and ultimately only one receives US Food and Drug Administration approval [2]. The average time for this process is about 10 to 15 years and costs around $\$ 800$ million to $\$ 1$ billion. We and others have hypothesized that all of the aforementioned issues can be addressed by human diseasespecific in vitro microphysiological systems that reconstitute organ-level functions at the tissue level.

Advances in stem cell biology and microfluidic fabrication technology have established the foundation to develop organ-inspired high-throughput in vitro assays. A critical breakthrough in biology was the discovery of human induced pluripotent stem (iPS) cells [3,4], which can be used for disease modeling and drug toxicity screening. Additionally, human iPS cells can be continuously expanded in culture in an undifferentiated state and then differentiated into different lineages; for example, cardiomyocytes (CMs), hepatocytes, adipocytes, or neurons [5]. By creating physiologically relevant microenvironments in microfluidic devices and utilizing human iPS cells, it is now possible to establish different three-dimensional tissue models, which can be used as drug screening systems (Figure 1). Of these, heart and liver models are of utmost significance (see Table 1). Cardiotoxicity accounts for about one-third of pharmaceuticals withdrawn because of safety issues [6], and the liver is the major site for drug metabolism and profoundly impacts the final effect of the drug. In vitro dynamic physiological systems combining heart and liver tissue will thus make the drug discovery process more economic, predictable, and efficient. 


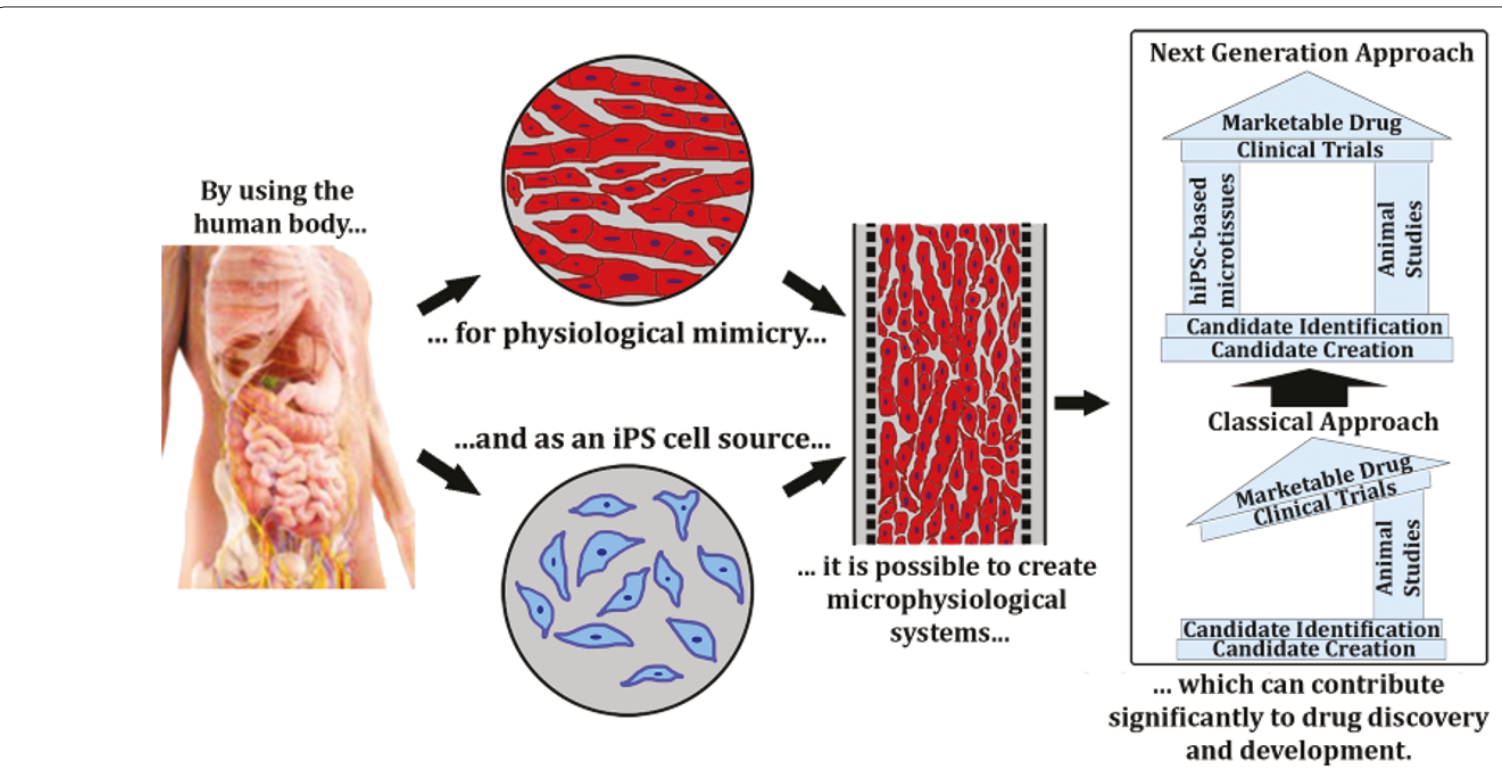

Figure 1. Microphysiological systems using human induced-pluripotent stem cell-derived microtissue. Microphysiological systems using induced pluripotent stem (iPS)-derived microtissue are able to provide an accurate physiological model for human tissue. Drug discovery and development can thus be drastically improved by introducing these systems to complement and fill gaps left by animal-based studies.

\section{Systems for high-content drug screening}

Classical cell culture systems based on multiwell culture dishes can in principle be used for drug testing, but are not amenable to physiological perfusion required by organ-like cultures. Although the integration of electrophysiological measurement capacity and advanced computational frameworks have enabled automatized and parallelized drug screening [6], multiwell culture systems are limited in terms of biomimicry. Using microfluidic technologies it is possible to create high-content drug screening systems, which simulate tissue structure and function at a micron level and bridge the gap between the in vivo and in vitro worlds. Bioinspired microfluidic environments allow spatiotemporal control of various chemical and physical culture conditions that are unavailable with other methods, which in turn allow for manufacturing of more physiologically relevant drug screening platforms. Microfluidic approaches also offer a number of advances, including a high degree of parallelization, miniaturization of large systems for convenient operation and reduction of reagent use, and unprecedented control of system architecture and dimensions $[7,8]$. Furthermore, because fluid flow in microfluidic channels is laminar, it can be mathematically modeled to make theoretical predictions of complex biological problems. These mathematical models coupled with experimental analysis provide a robust system for understanding the physiological complexity of micro-organ function in vitro, and facilitate promising approaches for addressing many biological problems, including organon-chip arrays for drug screening and disease modeling.
In recent years, microfluidic systems and microdevices have been employed for different purposes involving CMs and hepatocytes. A recent study employed a microfluidic system featuring hydrogel-coated channels to culture primary rat CMs in two dimensions [9]. Other applications included the modeling of specific conditions, such as ischemia/hypoxia [10]. Lee and coworkers have designed and tested an artificial liver sinusoid that mimics the three-dimensional organization of hepatocytes and employs an endothelial-like barrier to control the diffusion of nutrients and drug delivery [11]. By improving cell-cell contact, the structure allowed better maintenance of high-density three-dimensional hepatocytes. Micropatterning of human hepatocytes with fibroblasts or other stromal cells has been reported to improve hepatocyte function and allow hepatocyte proliferation [12]. We propose that for high content drug screening it is critical to create three-dimensional tissue constructs from normal and disease-specific human iPS cells to obtain faithful in vitro models that are predictive of in vivo conditions.

\section{Tissue models of the myocardium and liver}

To generate engineered three-dimensional tissue constructs in vitro, several different methods have been proposed in the literature. In the case of heart tissue, the most proposed three-dimensional engineered heart tissue consists of natural-derived materials such as collagen and Matrigel ${ }^{\mathrm{Tm}}$ with $\mathrm{CMs}$, and in some cases fibroblasts and vascular endothelial cells embedded in natural materials such as collagen and Matrigel ${ }^{\mathrm{mm}}$ [13-15]. 
Table 1. Examples of drugs approved for sale subsequently withdrawn from the market due to liver and cardiac toxicity

\begin{tabular}{lll}
\hline Drug & Year & Reason for withdrawal \\
\hline Terfenadine (Seldane) & 1998 & Risk of cardiac arrhythmias \\
Alpidem (Ananxyl) & 1999 & Rare serious hepatotoxicity \\
Grepafloxacin (Raxar) & 1999 & Prolonged QT interval \\
Troglitazone (Rezulin) & 2000 & Risk of hepatotoxicity \\
Trovafloxacin (Trovan) & 2001 & Risk of liver failure \\
Rofecoxib (Vioxx) & 2004 & Risk of myocardial infarction \\
Rosiglitazone (Avandia) & 2010 & Withdrawn in Europe because of increased risk of myocardial infarction and death. \\
& & This drug continues to be available in the USA \\
\hline
\end{tabular}

While these tissues physically contract in vitro, there is no proper organization of the cardiac cells. To address this lack of organization a few groups have placed neonatal rat CMs onto electrospun-aligned fibers and showed that alignment improved anisotropic electrophysiological properties as compared with monolayers or unorganized CMs [16,17]. Although electrospinning permits fabrication of aligned fibers, the low porosity limits cell infiltration and does not create threedimensional tissues, hence the resulting aligned tissues are thin monolayers. Recently, one group has employed mouse embryonic stem cell-derived CMs and neonatal rat ventricular CMs to make a two-dimensional anisotropic cardiac muscle tissue by microfabrication of fibronectin layers $[18,19]$. Combining this patterning method with cantilever-type microchips, these researchers were also able to quantify the exerted forces [20]. Yet, while they successfully showed that CMs aligned in the two-dimensional structure and generated contractile force, the model is not scalable to generate a threedimensional thick volume tissue. Similarly, nanoscale topography in two dimensions has been shown to affect anisotropic action potential propagation and tissue contractility of CM monolayers [21].

In the case of previously published efforts to create an in vitro liver model, similar issues and limitations apply. The liver is a vital organ for metabolism and detoxification. To develop an in vitro liver model for drug screening, recapitulation of hepatocyte polarity and function and viability have been of main interest, and various approaches toward achieving these goals included cell-matrix interactions, cell culture format, extracellular mechanical properties and mass transport. The sandwich culture, where monolayered hepatocytes are placed between two layers of matrix such as collagen or Matrigel ${ }^{\mathrm{Tm}}$, shows a cellular polarity in response to the extracellular environment and improved hepatic function and viability. For high-throughput screening applications, efforts are underway to achieve three-dimensional culture of hepatocytes. For example, hepatocyte spheroids were constructed in suspension in a high-throughput manner
[22], which maintained a more differentiated state as compared with monolayer culture. Three-dimensional hydrogels also have been extensively used to mimic the native liver niche (for example, porosity, mechanical modulus) and thus improve functionality and viability of the liver tissue construct in vitro [23]. In addition, extracellular matrix materials have been proven useful for encapsulating hepatocytes for the construction of threedimensional liver tissues in vitro. Collagen and glycosaminoglyans are the main extracellular matrix components of the human livers, and provide mechanical integrity to the liver tissues and present several bioactive signals to cells [24]. The absence of sufficient interactions with the extracellular matrix often causes cells to rapidly de-differentiate and die [25]. Furthermore, when engineering artificial cellular environments, it is important to provide hepatocytes with quantities and qualities of oxygen and nutrients similar to the highly vascularized native liver [26-28].

In summary, most of the current in vitro cardiac and liver models are either two-dimensional aligned structures, three-dimensional nonaligned models, or not scalable to high-throughput drug screening. Consequently, an artificial environment able to support a functional three-dimensional myocardium and liver within a microdevice is one of the critical steps for effective and efficient drug screening.

\section{Future outlook and challenges}

The urgent need for in vitro microphysiological systems has led to a steep growth of this intriguing new field, and many promising approaches and inventive systems have been presented in recent years. However, there are several challenges. First, selecting the right cell source for experiments is critical. Most studies to date are based on neonatal rodent-derived CMs, which do not mimic the human CMs due to metabolic differences between humans and animals. Clearly, systems using cells that represent human cardiac tissue are required. Recent developments in differentiating CMs from human iPS cells open the possibility of obtaining such human CMs 
in the laboratory [29]. For iPS-derived hepatocytes, it is important to establish robust differentiation methods, which allow stable in vitro function, genomic stability, and scalability.

Second, integrating multiple micro-organs on a single chip with physiological conditions and scaling those micro-organs as per the laws of biology will be challenging. In the human body, organs are interconnected via the circulatory system, and a drug designed for the heart may have off-target toxicity in the liver. Integrated in vitro microphysiological systems of human cardiac and liver tissue will not only serve as parallelized cardiotoxicity and liver toxicity tests, but also capture off-target toxicity. However, integration is not straightforward because each organ will have to be scaled in accordance to its size and function, and we do not know the minimal organoid size that satisfactorily recapitulates the drug response of the native organ. Although there are multiple approaches to address these issues - for example, allometric scaling, scaling based on histological sections, or functional scaling - none has been validated using multiple organoids on a chip [30].

Finally, when successfully integrated, a universal culture medium that mimics blood will be required to maintain tissue viability and function. This medium should be able to transport various dissolved gases - for example, carbon dioxide and oxygen - and should provide all of the necessary nutrients, growth factors, and proteins in a physiologically relevant manner to the integrated tissue system.

In summary, microengineered three-dimensional in vitro platforms for high-content drug screening for therapeutic and toxic effects have a realistic potential to reduce reliance on animal models and complement, if not to ultimately replace animal studies. The success, however, will depend on how effectively the engineering and the biology can be integrated to create a clinically relevant in vitro organ system.

Abbreviations

CM, cardiomyocyte; iPS, induced pluripotent stem.

\section{Competing interests}

The authors declare that they have no competing interests.

\section{Acknowledgments}

The work, and publication of this article, was supported by UH2TR000487 and the National Institutes of Health Common Fund for the Microphysiological Systems Initiative.

\section{Declarations}

Publication of this supplement has not been supported by sponsorship. Articles have undergone the journal's standard review process. The Editors declare that they have no competing interests.

This article has been published as part of Stem Cell Research \& Therapy Volume 4 Supplement 1, 2013: Stem cells on bioengineered microphysiological platforms for disease modeling and drug testing. The full contents of the supplement are available online at http://www.stemcellres.com/supplements/4/S1.

\section{Author details}

'Department of Bioengineering, B131 Stanley Hall, University of California at Berkeley, Berkeley, CA 94720-1762, USA. ²Department of Materials Science and Engineering, 370 Hearst Memorial Mining Bldg. \#1760, University of California at Berkeley, Berkeley, CA 94720-1760, USA. ${ }^{8}$ Department of Electrical Engineering and Computer Science, University of California at Berkeley, Berkeley, CA 94720, USA. ${ }^{3}$ Current address: School of Material Science \& Engineering, Gwangju Institute of Science and Technology, Oryong-dong, Buk-gu Gwangju 500-712, South Korea. ${ }^{4}$ Department of Surgery, Division of Transplantation and Eli and Edythe Broad Center of Regeneration Medicine and Stem Cell Research, University of California at San Francisco, San Francisco, CA 94143, USA. ${ }^{5}$ Gladstone Institute of Cardiovascular Disease, San Francisco, CA 94158, USA. ${ }^{6}$ Department of Medicine, Division of Genomic Medicine, University of California at San Francisco, San Francisco, CA 94143, USA. ${ }^{2}$ Liver Center, University of California at San Francisco, San Francisco, CA 94143, USA.

Published: 20 December 2013

References

1. US Food and Drug Administration [http://www.sourcewatch.org/index php/U.S._Food_and_Drug_Administration]

2. Wu MH, Huang SB, Lee GB: Microfluidic cell culture systems for drug research. Lab Chip 2010, 10:939-956.

3. Takahashi K, Tanabe K, Ohnuki M, Narita M, Ichisaka T, Tomoda K, Yamanaka S: Induction of pluripotent stem cells from adult human fibroblasts by defined factors. Cell 2007, 131:861-872.

4. Yu JY, Vodyanik MA, Smuga-Otto K, Antosiewicz-Bourget J, Frane JL, Tian S, Nie J, Jonsdottir GA, Ruotti V, Stewart R, Slukvin II, Thomson JA: Induced pluripotent stem cell lines derived from human somatic cells. Science 2007, 318:1917-1920.

5. Inoue H, Yamanaka S: The use of induced pluripotent stem cells in drug development. Clin Pharmacol Ther 2011, 89:655-661.

6. Guo L, Abrams RM, Babiarz JE, Cohen JD, Kameoka S, Sanders MJ, Chiao E, Kolaja KL: Estimating the risk of drug-induced proarrhythmia using human induced pluripotent stem cell-derived cardiomyocytes. Toxicol Sci 2011, 123:281-289.

7. Hung PJ, Lee PJ, Sabounchi P, Aghdam N, Lin R, Lee LP: A novel high aspect ratio microfluidic design to provide a stable and uniform microenvironment for cell growth in a high throughput mammalian cell culture array. Lab Chip 2005, 5:44-48.

8. Hung PJ, Lee PJ, Sabounchi P, Lin R, Lee LP: Continuous perfusion microfluidic cell culture array for high-throughput cell-based assays. Biotechnol Bioeng 2005, 89:1-8.

9. Annabi N, Selimović Š, Acevedo Cox JP, Ribas J, Afshar Bakooshli M, Heintze D, Weiss AS, Cropek D, Khademhosseini A: Hydrogel-coated microfluidic channels for cardiomyocyte culture. Lab Chip 2013, 13:3569-3577.

10. Ren L, Liu W, Wang Y, Wang J-C, Tu Q, Xu J, Liu R, Shen S-F, Wang J: Investigation of hypoxia-induced myocardial injury dynamics in a tissue interface mimicking microfluidic device. Anal Chem 2013, 85:235-244.

11. Lee PJ, Hung PJ, Lee LP: An artificial liver sinusoid with a microfluidic endothelial-like barrier for primary hepatocyte culture. Biotechnol Bioeng 2007, 97:1340-1346.

12. Khetani SR, Bhatia SN: Microscale culture of human liver cells for drug development. Nat Biotechno/ 2008, 26:120-126.

13. Eschenhagen T, Fink C, Remmers U, Scholz H, Wattchow J, Weil J, Zimmerman W, Dohmen HH, Schafer H, Bishopric N, Wakatsuki T, Elson EL: Threedimensional reconstitution of embryonic cardiomyocytes in a collagen matrix: a new heart muscle model system. Faseb J 1997, 11:683-694.

14. Song H, Yoon C, Kattman SJ, Dengler J, Masse S, Thavaratnam T, Gewarges M, Nanthakumar K, Rubart M, Keller GM, Radisic M, Zandstra PW: Interrogating functional integration between injected pluripotent stem cell-derived cells and surrogate cardiac tissue. Proc Natl Acad Sci U S A 2010, 107:3329-3334.

15. Zimmermann WH, Fink C, Kralisch D, Remmers U, Weil J, Eschenhagen T: Three-dimensional engineered heart tissue from neonatal rat cardiac myocytes. Biotechnol Bioeng 2000, 68:106-114.

16. Bursac N, Parker KK, Iravanian S, Tung L: Cardiomyocyte cultures with controlled macroscopic anisotropy - a model for functional electrophysiological studies of cardiac muscle. Circ Res 2002, 91:E45-E54

17. Zong XH, Bien H, Chung CY, Yin LH, Fang DF, Hsiao BS, Chu B, Entcheva E: Electrospun fine-textured scaffolds for heart tissue constructs. Biomaterials 
2005, 26:5330-5338.

18. Domian IJ, Chiravuri M, van der Meer P, Feinberg AW, Shi X, Shao Y, Wu SM, Parker KK, Chien KR: Generation of functional ventricular heart muscle from mouse ventricular progenitor cells. Science 2009, 326:426-429.

19. Feinberg AW, Feigel A, Shevkoplyas SS, Sheehy S, Whitesides GM, Parker KK: Muscular thin films for building actuators and powering devices. Science 2007, 317:1366-1370.

20. Grosberg A, Alford PW, McCain ML, Parker KK: Ensembles of engineered cardiac tissues for physiological and pharmacological study: heart on a chip. Lab Chip 2011, 11:4165-4173.

21. Kim DH, Lipke EA, Kim P, Cheong R, Thompson S, Delannoy M, Suh KY, Tung L, Levchenko A: Nanoscale cues regulate the structure and function of macroscopic cardiac tissue constructs. Proc Natl Acad Sci U S A 2010, 107:565-570.

22. Wu FJ, Friend JR, Lazar A, Mann HJ, Remmel RP, Cerra FB, Hu WS: Hollow fiber bioartificial liver utilizing collagen-entrapped porcine hepatocyte spheroids. Biotechnol Bioeng 1996, 52:34-44.

23. Kaufmann PM, Heimrath S, Kim BS, Mooney DJ: Highly porous polymer matrices as a three-dimensional culture system for hepatocytes. Cell Transplant 1997, 6:463-468.

24. Reid LM: Stem cell biology, hormone/matrix synergies and liver differentiation. Curr Opin Cell Biol 1990, 2:121-130.
25. Kulig KM, Vacanti JP: Hepatic tissue engineering. Transp/ Immuno/ 2004, 12:303-310.

26. Borenstein JT, Weinberg EJ, Orrick BK, Sundback C, Kaazempur-Mofrad MR, Vacanti JP: Microfabrication of three-dimensional engineered scaffolds. Tissue Eng 2007, 13:1837-1844

27. Chan C, Berthiaume F, Nath BD, Tilles AW, Toner M, Yarmush ML: Hepatic tissue engineering for adjunct and temporary liver support: critical technologies. Liver Transp/ 2004, 10:1331-1342.

28. Domansky K, Inman W, Serdy J, Dash A, Lim MHM, Griffith LG: Perfused multiwell plate for 3D liver tissue engineering. Lab Chip 2010, 10:51-58.

29. Lian XJ, Hsiao C, Wilson G, Zhu KX, Hazeltine LB, Azarin SM, Raval KK, Zhang $\mathrm{JH}$, Kamp TJ, Palecek SP: Robust cardiomyocyte differentiation from human pluripotent stem cells via temporal modulation of canonical Wnt signaling. Proc Natl Acad Sci U S A 2012, 109:E1848-E1857.

30. Wikswo JP, Curtis EL, Eagleton ZE, Evans BC, Kole A, Hofmeister LH, MatloffWJ: Scaling and systems biology for integrating multiple organs-on-a-chip. Lab Chip 2013, 13:3496-3511.

doi:10.1186/scrt375

Cite this article as: Mathur $A$, et al: Human induced pluripotent stem cellbased microphysiological tissue models of myocardium and liver for drug development. Stem Cell Research \& Therapy 2013, 4(Suppl 1):S14. 